\title{
Recursive Fuzzy Predictions of Future Patient Paths to Support Clinical Decision Making in ICU
}

\author{
A. Zeghbib ${ }^{1}$, M. Mahfouf ${ }^{1}$, J. J. Ross ${ }^{2}$, G. H. Mills ${ }^{2}$, G. Panoutsos ${ }^{1}$, M. Denai ${ }^{3}$, S. Suzani ${ }^{1}$ \\ ${ }^{1}$ Department of Automatic Control \& Systems Engineering, University of Sheffield, Sheffield, UK \\ ${ }^{2}$ Northern General Hospital, Sheffield, UK \\ ${ }^{3}$ Teesside University, Middlesbrough, UK \\ Email: a.zeghbib@sheffield.ac.uk
}

Received 2013

\begin{abstract}
In this paper, we propose a new architecture that combines prediction and decision-making in the form of a hybrid framework aimed at providing clinicians with transparent and accurate maps, or charts, to guide and to support treatment decisions, and to interrogate the clinical patients' course as it develops. These maps should be patient-specific, with options displayed of possible treatment pathways. They would suggest the optimal care pathways, and the shortest routes to the most efficient care, by predicting clinical progress, testing the ensuing suggestions against the developing clinical state and patient condition, and suggesting new options as necessary. These maps should also mine an extensive database of accumulated patient data, modelled diseases, and modelled patient-responses based on expert-derived rules. These individualized hierarchical targets, which are implemented in order to prevent life-threatening illnesses, will also have to "adapt" to the patient's altering clinical condition. Therapies that support one system can destabilize others and selecting which specific support to prioritize is an uncertain process, the prioritization of which can vary between clinical experts. Whilst clinical therapeutic decisions can be made with some degree of anticipation of the "likely" outcome (based on the experts' opinion and judgment), treatment is essentially rooted in the present, and is dependent on analyzing the current clinical condition and available data. The recursive learning approach presented in this paper, allows decision rules to predict the possible future course, and reflects back derived information from such projections to the present time and thus support proactive clinical care rather than reactive clinical care. The proposed framework for such a patient map supports and enables an optimized choice from available options and also ensures that decisions are based on both the available evidence and a database of best clinical practice. Preliminary results are encouraging and it is hoped to validate the approach clinically in the near future.
\end{abstract}

Keywords: Proactive Treatment, Clinical Decision, Intensive-Care, Patient-Paths, Physiological Map

\section{Introduction}

Preliminary studies on physiological patient state classification and patient map elaboration consists of: a) collecting patients' data from each subsystem, such as the heart, the lungs, the renal system, etc.; b) the Laboratory results; c) and medications. Such clinical information allows the identification of the current patient state, but does not provide information about the future patient state. [1,2] are study examples that consider the set of vital parameters from the cardio-vascular system (CVS) and respiratory system. For the respiratory and gas exchange systems there are many different models developed to deal with gas exchange in the lungs [3,4]. Hence, other approaches have been developed as simulators to describe the ventilation and the gas exchange interactions $[5,6]$. For a lumped system of arterial, tissue, venous and pulmonary compartments, the SOPAVent (Simulation of
Patient under Artificial Ventilation) model has also been developed to simulate the exchange of $\mathrm{O}_{2}$ and $\mathrm{CO}_{2}$ in the lungs and tissues together with their transport through the circulatory system $[7,8]$. Further improvements of the original, SOPAVent model, have also been included to develop a none-invasive model structure as well as a continuously updated model to improve patient-specific model performance and improve the prediction accuracy. A more recent study [9] combined a model of respiratory mechanics, a model of the human lung absolute resistivity and a 2-D finite-element mesh of the thorax to simulate EIT image reconstruction during mechanical ventilation.

The goals for treating critically-ill patients in Intensive Care Units are, of necessity, patient specific. A Clinical Decision Support System to optimize a patient's care would ideally have the following features: 1) function as 
a 'virtual Star Chamber' pooling all available expertise of the clinical staff in that Unit; 2) use this wealth of knowledge to derive current treatment for an individual patient via data bases which can ideally be interrogated; 3 ) use data-mining tools to categorize the current patient condition; 4) integrate current treatment protocols into real-time care; 5) exploit a predictive function to model and thus predict the clinical course of the patient into the future resulting from this care; 6) Test, "off-line", the consequences of the current treatment actions; 7) Finally, interrogate the generated data from the patient to check conformity with the predicted outcome

In the Intensive Care environment, clinical decisions are made to maintain patients' physiological parameters within acceptable (safe) ranges whilst treating or improving the underlying illness. Clinicians rely on their knowledge and experience to plan appropriate therapy rules. These are applied to the developing clinical condition and the outcomes revisited and alterations considered for implementation.

Selecting the most appropriate treatment package from differing options raises the possibility of potentially diverging and conflicting clinical decisions, or therapy rules, and that selecting one path will engender a developing and necessarily diverging clinical course for that patient. This dilemma requires that the choices should be clearly identified and that any model-based method must be able to predict future consequences for each choice. However, clinical decisions entail a degree of uncertainty and do not have a clearly mapped outcome for the consequences. If a clinician has a patient with a diagnosis " $\mathrm{D}$ ", the therapeutic choices can be $\mathrm{T} 1, \mathrm{~T} 2$, or $\mathrm{T} 3$. Therapy "T1" may induce a complication and cause the patient to deteriorate. Therapeutic decision "T2" may improve the state of the patient, or may not change it. "T3" may treat the diagnosis $\mathrm{D}$, but entail collateral damage in other organs. Thus the clinician faces considerable uncertainty. A map detailing clear future outcomes in all possibilities for patients' anticipated clinical recovery paths will be useful undoubtedly.

The decision-support map would provide clinicians predicted pathways for multiple possible patient- states, until the patient enters a final state of stability with normalized values for all monitored physiological parameters. This stable state would in effect represent, in terms of dynamic systems, the so called equilibrium state.

In the framework proposed in this paper, each generated node of the path displays two types of information. The first describes the current values of physiological parameters as concept variables, and the second describes the drug that causes these concept variables to evolve according a certain trend. The physiological parameters are calculated by reflecting the connective interactions of the variables within each node and between the nodes, which thus functions as part of a dynamical biological system. Thus, the interaction between clinical concepts should keep a stable equilibrium within time or continuous until reach the equilibrium cycle, depending on the initial patient state and the expert knowledge data-base.

The displayed options of possible treatment pathways support a clinical proactive decision making. This model has two principle components: the first component is the State Transition Predictor (STP), and the second is the Patient Paths Network (PPN). The first component is an expert knowledge data-base of the basic clinical rules for different possible patient states based on a number of clinical concepts to be observed. The number of clinical concepts is determined by a possibility that a clinical outcome would in effect take place. The remainder of this paper will be organized as follows: Section 2 will detail the "recursive" concept as well as the associated algorithms of the physiological patient map. Section 3 will explain how the cause-effect relationship within patient physiological parameters can be represented via a fuzzy cognitive map. Finally, conclusions relating to the proposed study together with future research directions will be given in Section 4.

\section{Physiological Patient Map}

In order to populate the physiological patient map, we reconceptualise clinical conditions from general terms such as "critical", "stable" "mild", etc.; via fuzzy linguistic terms describing the values of the physiological parameters. Each parameter is described in one of three fuzzy linguistic terms: Low $(L)$, Medium $(M)$, and High $(H)$. For m clinical concepts (i.e. physiological parameters), the nodes (i.e. states) of patient paths are expressed as follows:

Let us consider a vector of $m$ variables:

$$
\begin{aligned}
& y_{k}(t)\left\{y_{k, 1}, ? \cdot \cdot y_{k, m}\right\} \\
& y_{k, i=1, m}(\text { Fuzzy Linguistic Terms) } \\
& \in\{L, M, H\}, k \text { : state index }
\end{aligned}
$$

We argue here that there are two components (Modules) of our Physiological patient map: state transition predictor (STP) and patient path network (PPN), see "Figure 1". The next sections will expand on these two modules:

\subsection{State Transition Predictor (STP)}

The STP has two inputs, the first input is represented by clinical concepts, $y_{k}(t)$, and the second input is represented by drugs, i.e.

$$
x_{k}(t)=\left\{x_{k, 1}, \cdots x_{k, n}\right\}, n \text { : drug vector dimension }
$$


The outputs are the new clinical concepts formulated also via fuzzy linguistic terms based on the previous two vector inputs $x(t)$ and $y(t)$. Hence, the output vector will be as follows:

$$
Y_{k}(t+1)=F\left(x_{k}(t), y_{k}(t)\right) .
$$

Thus the input matrix $\left[x(t)^{\prime}, y(t)^{\prime}\right]$ generates a new output vector as follows:

$$
\begin{aligned}
& Y_{k}(t+1)=\left\{Y_{k, 1}, \cdots Y_{k, M j}\right\} ; \\
& Y_{k, j=1, M j}=\left\{y_{k, 1}, \cdots y_{k, m}\right\} .
\end{aligned}
$$

The dimension, $M j$, of the victor $Y_{k}(t+1)$ depends on the state index, $k$. The state transition predictor (STP) represents our data-base of expert knowledge.

\subsection{Patient Path Network (PPN)}

This module has the specific task of memorising all the predicted states from the initial recorded state until the final predicted stable state and builds the network of all the generated states. Furthermore, the simulation run of this module is completed to extract all possible outcome paths that the patient clinical state may follow, "Figure 2”.

The nodes of each path indicate the physiological parameter values in fuzzy linguistic terms and the drugs that should be administrated in order to reach the next transition state. Here we consider the recording of only four physiological parameters; (in an intensive care environment of a patient with cardio-respiratory system failure): Cardiac Output (CO) is low $(L)$, Mean Arterial Pressure (MAP) is low $(L)$, mean airway pressure $\left(\mathrm{PaO}_{2}\right)$
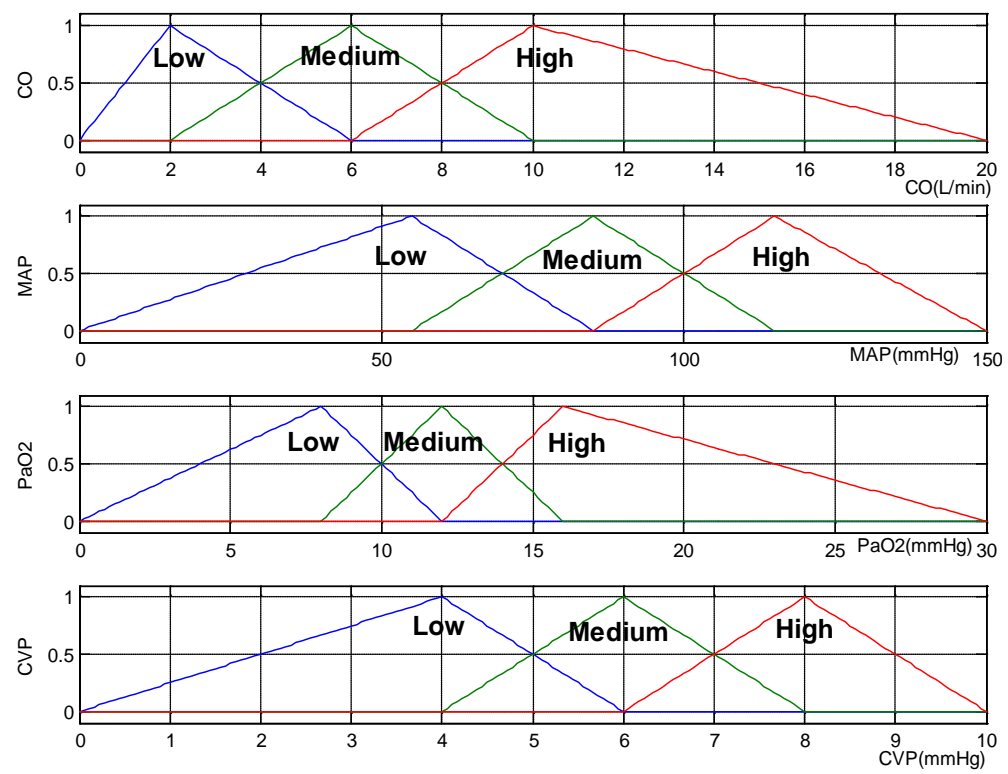

Figure 1. Fuzzy linguistic terms; Low, Medium, and High; of the four physiological parameters (clinical concepts) CO, MAP, $\mathrm{PaO}_{2}$, and $\mathrm{CVP}$. The $\mathrm{x}$-axis represents real values of these parameters.

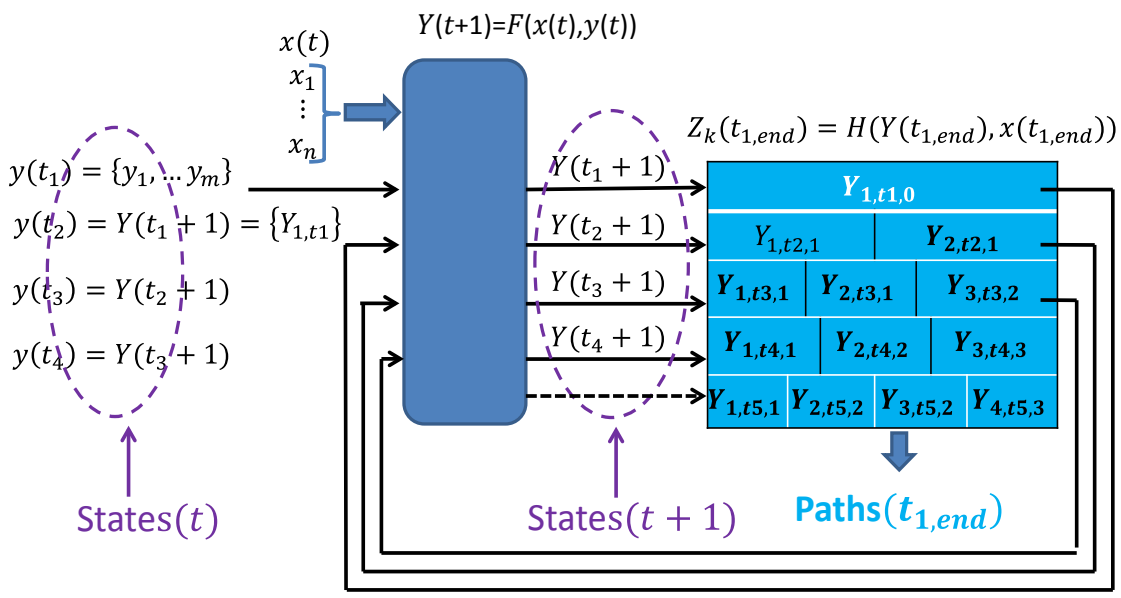

Figure 2. Physiological patient map (PPM). There are two modules. 
is low $(L)$, and Central Venous Pressure (CVP) is high $(H)$. Thus the recorded patient initial state was indicated in the model (see "Figure 3") as [LLLH]. The physiological patient map model in this case will generate the successive possible states of this patient in the form of paths network. The procedure is only stopped under two conditions: the first is when all the newly created states (nodes) reach the stable state [MMMM] or [HMMM], the second condition relates to when the newly state (node) has already been created, similarly to the case illustrated by "Figure 3", i.e. the state [MMMH].

Patient paths (trajectories) network nodes indicate the physiological parameter values in fuzzy linguistic terms and the drug input that should be administrated to reach the next transition state. There are five types of drugs used by the model to generate this paths-based network; $\mathrm{N}$ : noradrenaline, D: dobutamine, G: GTN, F: fluid, O: oxygen, and F: Fluid. These drugs are described also via two fuzzy linguistic terms: "p: positive" to increase the drug, and "l: less" to decrease the drug dosage. From the top-node of the initial recorded patient state, the model progresses recursively creating successor nodes and paths until reaching the equilibrium state of each patient path.

In the particular (realistic) example of "Figure 3", the patient map includes 12 possible physiological patient paths generated with this model. Section 3 will expand on the physiological parameters evolution within a specific path, in order to explain the successive nodes created from the model and their convergence to the sta- ble state (equilibrium).

\section{Physiological Parameters Evolution in Cognitive Map}

The cause-effect relationship within the patient physiological parameters is represented as a fuzzy cognitive map [10]. We argue that this graphical representation may be the ideal tool for reasoning with uncertainty.

The path nodes include " 9 " concepts; four " 4 " of which are causal concepts representing the patient physiological parameters. These concepts are, as is the case of in any dynamic system, in permanent interactions within causeeffect relationships. The valuations of these cause-effect relationships; from the experts; are carried-out indirectly through the drug concept values. The five " 5 " other concepts are drugs; their edges are directed only in one direction onto the physiological parameters, as illustrated in "Figure 4". These are the main considered variables for building the physiological patient map.

The predicted evolution of physiological parameters infers different possible patient trajectories in a clear physiological map. The illustration of one trajectory from the trajectories map of "Figure 4" can help to get to grips with the mechanism used by predicted clinical concepts interactions to build the successive future patient outcomes, as shown in "Figure 5". The model operates iteratively, because at each time interval the new concept node values are predicted using the previous node concept values.

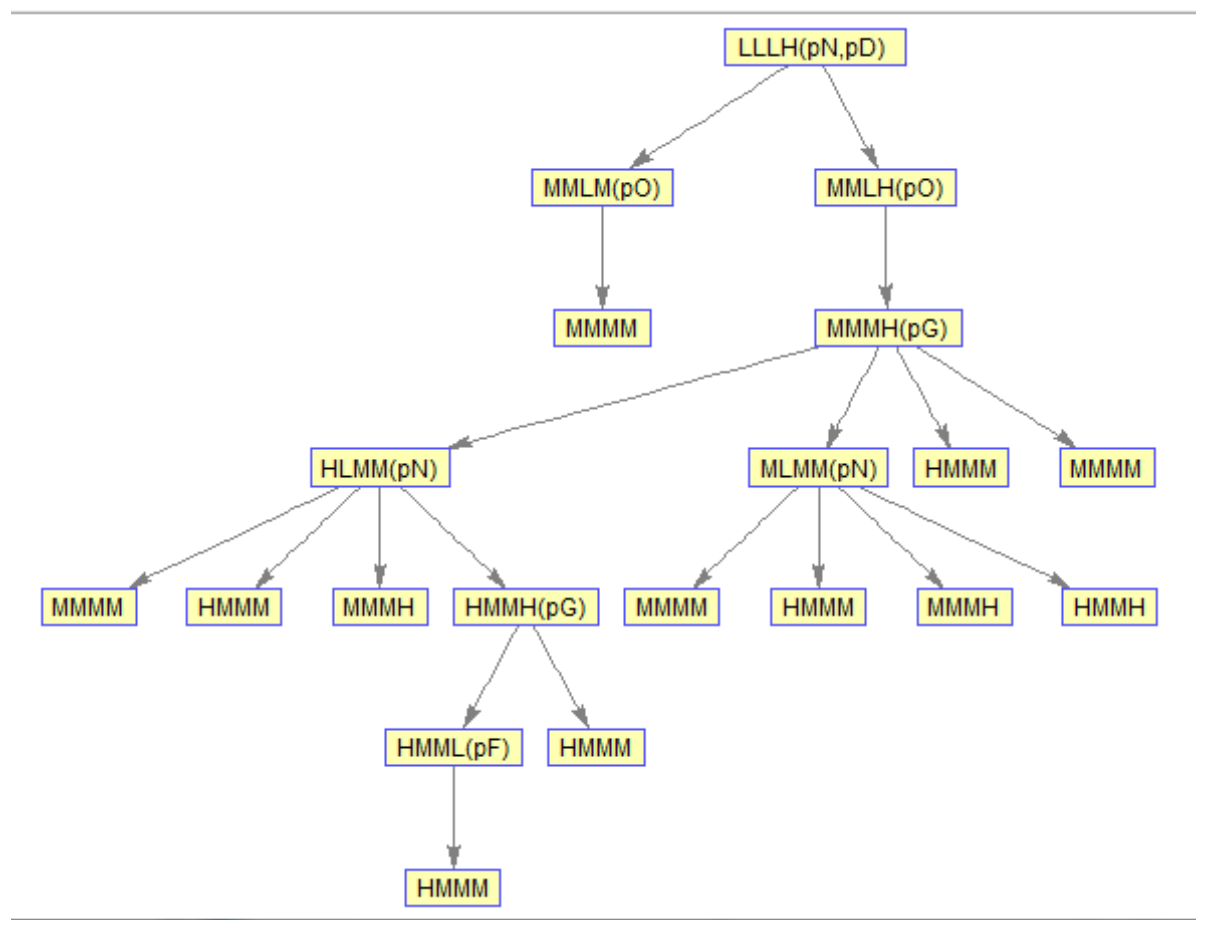

Figure 3. Network of physiological parameters and different possible patient paths prediction. 


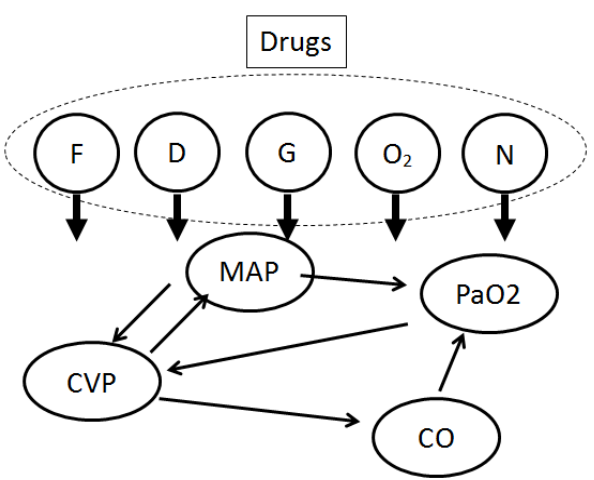

Figure 4. Fuzzy cognitive map, within each node of the patient path, for the cause-effect relationship of patient physiological parameters and drugs. The drugs have uniderictional effects. Drug types used in this study are; N: noradrenaline, D: dobutamine, G: GTN, F: fluid, $\mathrm{O}_{2}$ : oxygen, and F: Fluid. The map here uses node shapes relating to the concept type. Drug concepts are circles, physiological parameters are elipses.

\begin{tabular}{|c|c|c|c|c|c|c|c|c|}
\hline & DRUG & $\mathrm{pN}, \mathrm{pD}$ & po & $\mathrm{pG}$ & $\mathrm{pN}$ & $\mathrm{pG}$ & $\mathrm{pF}$ & \\
\hline \multirow{3}{*}{ CO } & L & $\mathrm{L}>$ & & & & & & \\
\hline & M & & $M$ & $\Rightarrow M_{1}$ & & & & \\
\hline & $\mathrm{H}$ & & & & $\triangle \mathrm{H}-$ & $\longrightarrow \mathrm{H}$ & $\Rightarrow \mathrm{H}$ & $\Rightarrow \mathrm{H}$ \\
\hline \multirow{3}{*}{ MAP } & L & L & & & $\pi^{\mathrm{L}}$ & & & \\
\hline & M & & $M$ & $\Rightarrow M$ & & $M$ & $\Rightarrow M$ & $\Rightarrow M$ \\
\hline & $\mathrm{H}$ & & & & & & & \\
\hline \multirow{3}{*}{$\mathrm{PaO2}$} & $\mathrm{L}$ & $\mathrm{L}-$ & $\rightarrow \mathrm{L}$ & & & & & \\
\hline & M & & & $\triangle_{M}$ & $\rightarrow M$ & $\Rightarrow M=$ & $\Rightarrow \mathrm{M}=$ & $\Rightarrow M$ \\
\hline & $\mathrm{H}$ & & & & & & & \\
\hline \multirow{3}{*}{ CVP } & $L$ & & & & & & $a^{L}>$ & \\
\hline & $M$ & & & & ${ }^{M}$ & & & $-M$ \\
\hline & $\mathrm{H}$ & $\mathrm{H}-$ & $\Rightarrow \mathrm{H}$ & $\Rightarrow \mathrm{H}$ & & $\Delta_{\mathrm{H}}$ & & \\
\hline
\end{tabular}

Figure 5. Patient path evolution from the physiological map. This path has seven "7" physiological states (nodes). The initial state is [LLLH] and the outcome state is [HMMM], which is the final patient stable state. We observe the continous physiological parameters evolution in the same time, parallel way, until the system reaches its equilibrium state.

The path chosen from the map of "Figure 3" includes seven "7" physiological states (nodes), the initial state being [LLLH] and the outcome state [HMMM] being the final predicted patient stable state. In this path all physiological variables change continuously until the system reaches its equilibrium state. The convergence of a physiological dynamic system towards a stable state is only possible because of the available expert knowledge relating to the biological system behaviour. However, these paths can also diverge and it may be possible that they will never reach the "equilibrium" state.

\section{Conclusion}

The proposed physiological patient map model as detailed in this study has many advantages for the clinicians to deal with patients in intensive care unit. Usually, clin- ical decisions are based on clinical assessment and subjective judgment of clinicians. The clinicians' judgments are only reactive actions because the usual existent systems of assessment cannot provide the possible outcome set of the future scenarios of physiological patient states. This model provides the clinicians transparent map, based on their expert-knowledge, of future patient paths that will be conveyed back to the current planning context in order to support proactive clinical actions rather than just reactive actions. This map allows, in terms of professional accountability, a real choice of the best decision and also ensures that decisions are based on available evidence. However as this model is based on the fuzzy sets of clinical concepts, the increase of recorded physiological parameters and drugs will make the physiological patient map more complex and also raises the patient paths number but it is believed that as long as the map itself is systematically built, accuracy and most importantly transparency will still be conserved. Immediate future plans will include validation of this map concept on a simulated patient platform, but our "not so immediate" research plan will include two vectors: the first one is to introduce variations of the physiological parameters to test the generalization properties of the overall framework as to its quality of control and to path convergence; the second will provide this model with new tools using probability and possibility theories both for control and path selection. Both data and knowledge fusion, especially in the case of information conflicts, will also be a pertinent issue to resolve.

\section{Acknowledgements}

The authors wish to acknowledge financial support for this work from the UK-EPSRC under Grant EP/FO2889X/l.

\section{REFERENCES}

[1] J. J. Ross, M. A. Denai and M. Mahfouf, “A Hybrid Hierarchical Decision Support System for Cardiac Surgical Intensive Care Patients, Part II-Clinical Implementation and Evaluation,” Artificial Intelligence in Medicine, Vol. 45, No. 1, 2009, pp. 53-62.

http://dx.doi.org/10.1016/j.artmed.2008.11.010

[2] M. A. Denai, M. Mahfouf and J. J. Ross, “A Hybrid Hierarchical Decision Support System for Cardiac Surgical Intensive Care Patients, Part I-Physiological Modelling and Decision Support Design,” Artificial Intelligence in Medicine, Vol. 45, No. 1, 2009, pp. 35-52. http://dx.doi.org/10.1016/j.artmed.2008.11.009

[3] T. Winkler and A. Krause, "Simulation of Mechanical Respiration Using a Multi-Compartment Model for Ventilation Mechanics and Gas Exchange," Journal of Clinical Monitoring and Computing, Vol. 12, 1995, pp. 231239. http://dx.doi.org/10.1007/BF01207204

[4] A. Ben-Tal, "Simplified Models for Gas Exchange in the 
Human Lungs," International Journal of Evolutionary Biology, Vol. 238, 2006, pp. 474-495. http://dx.doi.org/10.1016/j.jtbi.2005.06.005

[5] C. J. Hinds, M. J. Roberts, D. Ingram and C. J. Dickinson, "Computer Simulation to Predict Patient Responses to Alterations in the Ventilation Regime," Intensive Care Medicine, Vol. 22, 1984, pp. 13-22.

[6] J. G. Hardman, N. M. Bedforth, A. B. Ahmed, R. P. Mahajan and A. R. Aitkenhead, "A Physiology Simulator: Validation of Its Respiratory Components and Its Ability to Predict the Patient's Response to Changes in Mechanical Ventilation,” British Journal of Anaesthesia, Vol. 81, No. 3, 1998, pp. 327-332.

http://dx.doi.org/10.1093/bja/81.3.327

[7] A. Wang, M. Mahfouf, G. H. Mills, G. Panoutsos, D. A. Linkens, K. Goode, H.-F. Kwok and M. Denaï, "Intelligent Model-Based Advisory System for the Management of Ventilated Intensive Care Patients-Part I: Hybrid Blood Gas Patient Model," Computer Methods and Programs in Biomedicine, Vol. 99, No. 2, 2010, pp. 195-207. http://dx.doi.org/10.1016/j.cmpb.2009.09.011

[8] A. Wang, M. Mahfouf, G. H. Mills, G. Panoutsos, D. A. Linkens, K. Goode, H.-F. Kwok and M. Denaï, "Intelligent Model-Based Advisory System for the Management of Ventilated Intensive Care Patients-Part II: Decision Support," Computer Methods and Programs in Biomedicine, Vol. 99, No. 2, 2010, pp. 208-217. http://dx.doi.org/10.1016/j.cmpb.2010.03.009

[9] M. A. Denai, M. Mahfouf, S. Mohamed Samuri, G. Panoutsos, B. H. Brown and G. H. Mills, "Absolute Electrical Impedance Tomography (aEIT) Guided Ventilation Therapy in Critical Care Patients: Simulations and Future Trends," IEEE Transactions on Information Technology in Biomedicine, Vol. 14, No. 3, Special Issue "New and Emerging Technologies in Bioinformatics and Bioengineering”, 2010.

[10] B. Kosko, “Fuzzy Cognitive Maps,” International Journal of Man-Machine Studies, Vol. 24, 1986, pp. 65-75. http://dx.doi.org/10.1016/S0020-7373(86)80040-2 